\title{
Correction to: Effects of grammar complexity on artificial grammar learning
}

\author{
Esther Van den Bos ${ }^{1}$ • Fenna H. Poletiek ${ }^{1}$ \\ Published online: 18 June 2019 \\ (C) The Psychonomic Society, Inc. 2019
}

\section{Correction to: Mem Cogn https://doi.org/10.3758/MC.36.6.1122}

In the article "Effects of Grammar Complexity on Artificial Grammar Learning" by E. Van den Bos and F. Poletiek, published in Memory \& Cognition, 2008, 36(6), 1122-1131, doi:10.3758/MC.36.6.1122, an error was made in the computation of topological entropy (TE). A transition between $n$ grams was coded on the basis of one overlapping letter instead of $n-1$ overlapping letters (e.g., in Grammar B, transitions were coded from _ ZT to TPR and TPQ instead of to ZTP). This led to an inflation of transitions in grammars described by larger $n$-grams (i.e., lifted more than once; Bollt \& Jones, 2000), and hence TE. Corrected values are reported in Table 1.

The article examined two aspects of complexity: the number of associations and the length of the associations that have to be learned. Corrected TE is no longer correlated with the number of elements required to predict the next (i.e., the length of the associations, NE, $r=.53, p=.113$ ), but highly correlated with the number of bigrams and the number of rules (i.e., the number of associations, $r=.99, p<.001$, for both). The regression analyses were redone with corrected $\mathrm{TE}$ as a measure of the number of associations and NE as a measure of the length of the associations. The pattern of results was similar to that reported in the paper.

The online version of the original article can be found at https://doi.org/ 10.3758/MC.36.6.1122

Esther Van den Bos

bosejvanden@fsw.leidenuniv.nl

1 Institute of Psychology, Leiden University, PO Box 9555, 2300 RB Leiden, The Netherlands
$\mathrm{NE}$ was the best predictor of the overall proportion of correct classifications in the memorize condition. The model including only TE accounted for $13.2 \%$ of the variance, $F(1,28)$ $=4.27, p=.048, \beta=-.364$. The model including only $\mathrm{NE}$ accounted for $15.7 \%$ of the variance, $F(1,28)=5.21, p=.030$, $\beta=-.396$. Adding TE was no significant improvement to the model including only NE, $F_{\text {change }}(1,27)=1.09, p=.306$. In the look for rules condition, all models remained nonsignificant.

In the analyses by type of test string, the model including only NE was significant for strings containing two or more second-order violations in the memorize condition, $F(1,22)=$ 6.02, $p=.023, \beta=-.463$. In the look for rules condition, the model including only NE was significant for strings containing three first-order violations, $F(1,22)=7.13, p=.014, \beta=.495$. The models including TE no longer reached significance: TE only, $F(1,22)=1.03, p=.320$; TE and NE, $F(2,21)=3.41, p=$ .052. All other models remained nonsignificant.

Our conclusions that implicit learning of artificial grammars is affected by complexity and that the length of the dependencies that have to be acquired seems the most influential aspect of complexity remain valid. However, topological entropy should not be considered a sensitive measure of this aspect. 
Table 1 Values on four measures of complexity by grammar

\begin{tabular}{lllll}
\hline Grammar & NR & NB & NE & Corrected TE \\
\hline A & 20 & 30 & 2 & 0.5543 \\
B & 21 & 33 & 3 & 0.6019 \\
C & 22 & 36 & 3 & 0.6753 \\
D & 22 & 36 & 4 & 0.6823 \\
E & 23 & 38 & 2 & 0.7131 \\
F & 24 & 41 & 4 & 0.7465 \\
G & 24 & 40 & 3 & 0.7586 \\
H & 25 & 44 & 4 & 0.8021 \\
I & 26 & 45 & 3 & 0.8449 \\
J & 27 & 47 & 4 & 0.8587 \\
\hline
\end{tabular}

$\mathrm{NR}=$ number of rules; $\mathrm{NB}=$ number of bigrams; $\mathrm{NE}=$ number of elements required to predict the next; corrected $\mathrm{TE}=$ corrected topological entropy
Open practices statement The data and materials for the experiment are available upon request: https://hdl.handle.net/10411/EATY2Z. The experiment was not preregistered.

\section{Reference}

Bollt, E. M., \& Jones, M. A. (2000). The complexity of artificial grammars. Nonlinear Dynamics, Psychology, and Life Sciences, 4, 153168.

Publisher's note Springer Nature remains neutral with regard to jurisdictional claims in published maps and institutional affiliations. 